\title{
MITHRA - multiparametric MR/CT image adapted brachytherapy (MR/CT-IABT) in anal canal cancer: a feasibility study
}

\author{
Luca Tagliaferri, MD!', Stefania Manfrida, MD!, Prof. Brunella Barbaro, MD², Maria Maddalena Colangione, MD! \\ Valeria Masiello, MD!, Prof. Gian Carlo Mattiucci, MD!, Elisa Placidi, MD³, Rosa Autorino, MD! \\ Prof. Maria Antonietta Gambacorta, MD', Silvia Chiesa, MD', Prof. Giovanna Mantini, MD', \\ Prof. György Kovács, MD, PhD4, Prof. Vincenzo Valentini, MD \\ IRadiation Oncology Department, Gemelli-ART, Università Cattolica del Sacro Cuore, Rome, Italy, ²Department of Radiological Sciences, \\ Università Cattolica del Sacro Cuore, Rome, Italy, ${ }^{3}$ Physics Institute, Università Cattolica del Sacro Cuore, Rome, Italy, ${ }^{4}$ Interdisciplinary \\ Brachytherapy Unit, University of Lübeck \& University Hospital S-H, Campus Lübeck, Germany
}

\begin{abstract}
Purpose: The aim of this study is to test a novel multiparametric imaging guided procedure for high-doserate brachytherapy in anal canal cancer, in order to evaluate the feasibility and safety.

Material and methods: For this analysis, we considered all consecutive patients who underwent magnetic resonance/computed tomography image adapted brachytherapy (MR/CT-IABT) treated from February 2012 to July 2014. To conduct this project, we formed a working group that established the procedure and identified the indicators and benchmarks to evaluate the feasibility and safety. We considered the procedure acceptable if $90 \%$ of the indicators were consistent with the benchmarks. Magnetic resonance imaging with contrast and diffusion weighted imaging were performed with an MRI-compatible dummy applicator in the anus to define the position of the clinical target volume disease and biological information. A pre-implantation treatment planning was created in order to get information on the optimal position of the needles. Afterwards, the patient underwent a simulation CT and the definite post-implantation treatment planning was created.

Results: We treated 11 patients (4 men and 7 women) with MR/CT-IABT and we performed a total of 13 procedures. The analysis of indicators for procedure evaluation showed that all indicators were in agreement with the benchmark. The dosimetric analysis resulted in a median of $\mathrm{V}_{200}, \mathrm{~V}_{150}, \mathrm{~V}_{100}, \mathrm{~V}_{90}, \mathrm{~V}_{85}$, respectively of $24.6 \%, 53.4 \%$, $93.5 \%, 97.6 \%$, and $98.7 \%$. The median coverage index (CI) was 0.94 , the median dose homogeneity index (DHI) was 0.43 , the median dose non-uniformity ratio (DNR) resulted 0.56 , the median overdose volume index (ODI) was 0.27. We observed no episodes of common severe acute toxicities.

Conclusions: Brachytherapy is a possible option in anal cancer radiotherapy to perform the boost to complete external beam radiotherapy (EBRT). Magnetic resonance can also have biological advantages compared to the US. Our results suggest that the multiparametric MR/CT-IABT for anal cancer is feasible and safe. This new approach paves the way to prospective comparison studies between MRI and ultrasound-guided brachytherapy (USBT) in anal canal cancer.
\end{abstract}

J Contemp Brachytherapy 2015; 7, 5: 336-345 DOI: $10.5114 / j \mathrm{cb} .2015 .55118$

Key words: anal canal cancer, anal carcinoma, brachytherapy, MRI-guided brachytherapy, image adapted brachytherapy (IABT).

\section{Purpose}

Anal canal cancer is an uncommon disease, accounting for $1-2 \%$ of the tumors involving the digestive tract and affecting approximately 4300 patients per year in Europe [1]. External beam radiotherapy (EBRT) along with concomitant chemotherapy, resulting in organ preservation, is the first-line standard treatment in anal cancer $[2,3]$. Moreover, a boost to the tumor after 45 Gy EBRT +/- chemotherapy is used to achieve a good local control in high-risk patients. There is no agreement on the boost technique [4]. Intensity modulated radiotherapy (IMRT) [5, 6, 7] and brachytherapy (BT) are common therapeutic options for delivering local dose escalation. Nowadays, the combination of new
Address for correspondence: Luca Tagliaferri, MD, Dipartimento Scienze Radiologiche, Divisione di Radioterapia, Università Cattolica del Sacro Cuore, Largo A. Gemelli, 8 - 00168 Rome, Italy, phone: +39 0630154434, fax: +39 0630155908, „ e-mail: luca.tagliaferri@rm.unicatt.it
Received: 18.07.2015

Accepted: 25.09.2015

Published: 31.10 .2015 
technologies and techniques in the field of brachytherapy, such as intensity modulated brachytherapy (IMBT) and image guided brachytherapy (IGBT), has led to the use of image adapted brachytherapy (IABT), allowing clinicians to deliver higher doses to the tumor, and to reduce the dose to the surrounding normal tissue [8]. The most widely used imaging method for exact guidance of interstitial radiotherapy in anal cancer BT is endo-anal ultrasound (EAUS) [9] because of good target delineation and a very easy administration [8]. On the other hand, magnetic resonance imaging (MRI) is the gold standard imaging in staging and treatment response evaluation. Indeed, MRI provides excellent contrast and spatial resolution, providing information on tumor size, local extent and spreading, invasion of adjacent organs, and more accurate nodal involvement $[1,10]$. Furthermore, due to the use of perfusion and diffusion weighted imaging (DWI), MRI can also provide information concerning metabolic characteristics and microcirculation of the tumor [11]. These features could be useful for the modulation of the target dose, and consequently, the improvement of the treatment results. MRI-guided BT has already been used in other cancer sites, for example it has proven as the best solution for boost in cervical cancer treatment [12].

The aim of this study was to test a novel multiparametric imaging (MR/CT) guided procedure for high-dose-rate (HDR) brachytherapy (MR/CT-image adapted brachytherapy - MR/CT-IABT) in anal canal cancer, in order to evaluate its feasibility and safety.

\section{Material and methods}

\section{Working group (MITHRA - WG)}

In order to assess the feasibility of this new procedure, we formed an interdisciplinary team including a radiation oncology expert in $\mathrm{BT}$, a radiation oncology expert in treatment planning and image fusion, a radiology expert in MRI anal cancer, and a medical physicist expert in HDR interstitial brachytherapy. Moreover, we involved another radiation oncology expert in BT from another institution for external reviewing. The WG established the patient selection, procedure, and identified indicators and benchmarks for evaluating feasibility and safety.

\section{Patient selection}

In this paper, we considered all consecutive patients who underwent a boost with MR/CT-IABT treated in the Catholic University of Rome. All data were retrieved from the hospital multidivisional electronic database - "Spider's Net" (Opengraph $\left.{ }^{\circledR}\right)$ [13]. Inclusion criteria were histologically proven diagnosis of primary anal canal cancer, tumor of any $\mathrm{T}$ stage and $\mathrm{N}$ stage, according to the $7^{\text {th }}$ edition of the TNM classification, absence of diagnosed distant metastases, informed consent, general good conditions with Karnofsky index $(>80)$. After EBRT treatment for primary tumor, the boost with MR/CT-IABT was administered only in case of local residues or $\mathrm{T} 4$ at the initial stage.
The reasons for exclusion were as follow: when the insertion of the applicator in the anal canal was impossible; infiltration of the rectum $(>12-13 \mathrm{~cm}$ cranial from the anal margin); if the clinical target volume (CTV) represented more than half of the anus circumference; patients with psychiatric disorders and with MRI contraindications. Also, cases with progressive disease after radiochemotherapy (RTCT) were excluded. All patients had a clinically detected disease by anorectal palpation, pelvic MRI and PET/CT.

\section{External beam radiotherapy and concomitant chemotherapy}

The radiochemotherapy protocol used in our Institute at the time of treatment prescription included 5 -FU (1000 mg/mq on days 1-4 and days 29-32) +/- mitomycin C (10 mg/mq on day 1 and day 29), associated with conventional 3D conformal radiotherapy (3DCRT), or after 2012, with intensity modulated radiotherapy (IMRT). Before 2012, the patients underwent an EBRT treatment with a split of 19 days. Radiotherapy was administered on the anal canal, the tumor (CTV1), and regional pelvic nodes, including the inguinal lymph nodes (CTV2).

The total dose to CTV1 was based on the protocol: 1) 3D EBRT treatment with a split: $58.5 \mathrm{~Gy}(1.8 \mathrm{~Gy} /$ day + concomitant boost of 90 cGy x 2 days/weeks); 2) 3D EBRT treatment without a split: 45 Gy (1.8 Gy/day); 3) IMRT treatment: 50 Gy (2 Gy/day) for T2, 55 Gy (2.2 Gy/day) for T3 or T4.

\section{Brachytherapy}

\section{Timing}

Four to six weeks after the end of the RTCT, patients underwent clinical and imaging (MRI) re-evaluation, and in case of local residues or a T4 at the initial stage, we administered an MR/CT-IABT.

\section{Pre-treatment procedure}

We programmed a pre-planning phase to obtain an optimal treatment. In fact, while ultrasound-guided brachytherapy (USBT) allows on-line evaluation, MRI still needs off-line conformal registration with CT-simulation. Patients underwent planning MRI study with an MRI-compatible dummy applicator [14]. Magnetic resonance imaging with contrast and diffusion weighted imaging (DWI) were performed to define the position of the scar or the residual disease. We acquired MR imaging data on 1.5-T unit (Horizon Advantage, GE Medical Systems, Milwaukee, WIS, USA). Nodular residual "tumor" tissues, intermediate or high tumor signal intensity (higher than muscle) were defined as residual tumor, on conventional T2 weighted-MRI sequences. The scar was defined as diffuse hypointense "fibrotic" thickening of the anal canal wall. On the DWI-MRI sequences ( $b$ factor $=1000 \mathrm{sec} / \mathrm{mm}^{2}$ ), the residual tumor was defined as a persistence of increased signal intensity on DWI in the previous tumor localization, using the normal rectal wall, outside the previous 
tumor area, as the internal reference. Three Regions of Interest (ROIs) were drawn on the dynamic T1-weighted spoiled gradient echo images section, taken from each section of the entire tumor, considering the corresponding T2-weighted axial images. ROI 1 involved the entire tumor area; ROI 2 involved the normal rectal wall adjacent to the tumor; ROI 3 only involved the high-contrast enhancement area within the tumor. ROI 3 was considered the residual tumor area. According to these data, the radiologist contoured the target based on the images (Figure 1).

At that point, patients underwent a CT-simulation with the applicator in situ. We used both the Martinez Universal Perineal Interstitial Template (MUPIT, Nucletron, an Elekta company, Elekta AB, Stockholm,

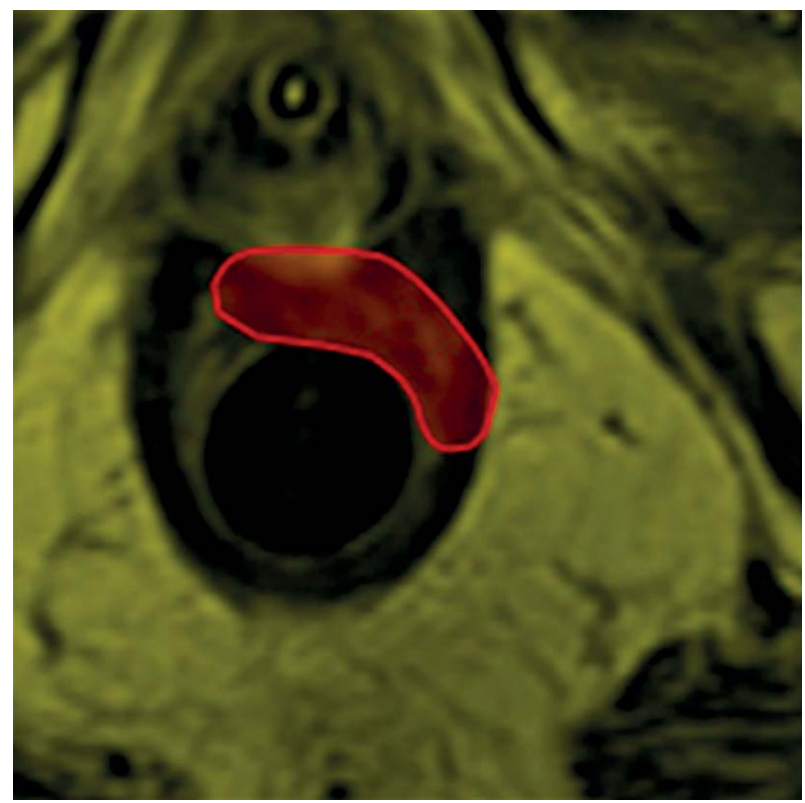

Fig. 1. Contouring with radiologist

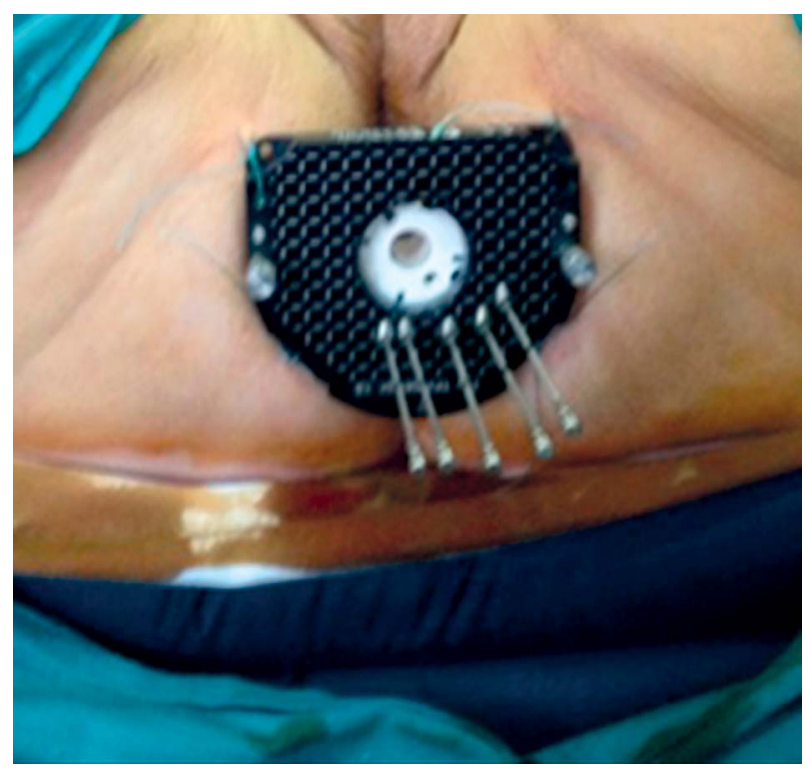

Fig. 3. Template Set Tps 081 (By Gfmmbh ${ }^{\circledR}$, Germany)
Sweden) (Figure 2) and the template set TPS 081 (by GfMmbH, Germany) (Figure 3). Simulation CT images were fused with the planning MRI images using rigid and deformable co-registration software Velocity $^{\circledR}$, Apache Software Foundation, USA). A pre-implantation treatment planning (pre-plan) was designed in order to retrieve information on the optimal position of the needles and the depth of insertion (Figure 4). We performed skin tattoos, to guarantee a correct repositioning of the template.

\section{Treatment}

An anesthetist administered spinal anesthesia and the patient was placed in the lithotomy position. Based

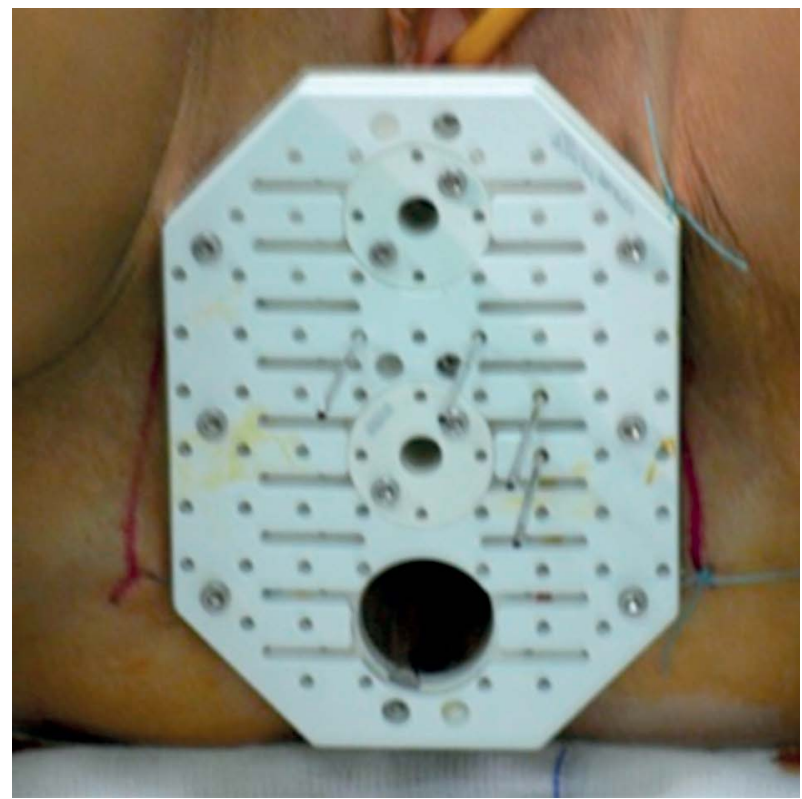

Fig. 2. MUPIT applicator $\left(\right.$ Elekta $\left.^{\circledR}\right)$

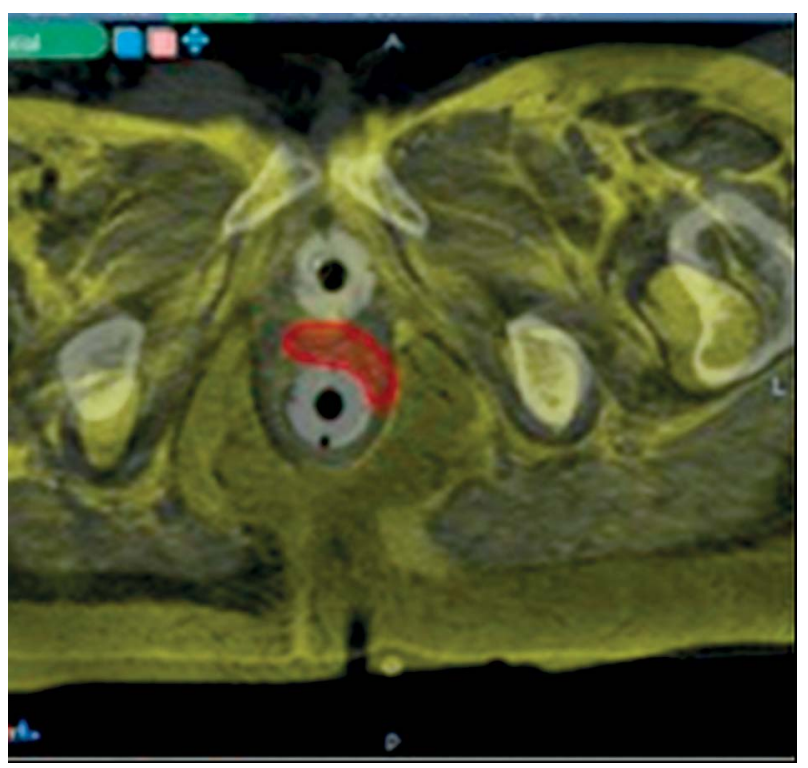

Fig. 4. Deformable co-registration (Velocity ${ }^{\circledR}$ ) 
on the skin tattoos, the template was inserted and fixed to the skin by sutures, and needles were positioned according to the pre-plan indications. Following needle implantation, the patient underwent a CT scan once again and a definite post-implantation treatment (postplan) was planned on the Oncentra Brachy (Nucletron, an Elekta company, Elekta AB, Stockholm, Sweden) console. Patients were maintained in the same position throughout the procedure with Zephir $^{\odot}$ patient transport system. The needle placing and dose planning followed the geometry standards of the Paris system with additional manual volume optimization. The CTV and dose on organs at risk (OARs) were documented using a dose-volume histogram (DVH). The CTV corresponded to residual tumor or scar, whereas OARs were the rectum and bladder. The boost dose was applied using a Microselectron Digital $^{\odot}$ HDR- BT device (Nucletron, an Elekta company, Elekta $A B$, Stockholm, Sweden) with a source of ${ }^{192} \mathrm{Ir}$ (370 GBq initial activity). The total dose was evaluated case by case, so as to reach a biological effective dose (BED) of about 70 Gy (total external radiation therapy [ERT] + BT) and an $\mathrm{EQD}_{2}$ of $60 \mathrm{~Gy}$. For this reason, the dose was calculated between 3.5 and 7 Gy prescribed on the actual CTV. When the BT dose exceeded 4 Gy, we delivered it in 2 fractions [9] with a protocol interval of 1 week, with the exception of patients treated with a split during the ERT.

\section{Dosimetric criteria}

We calculated the median and range of $\mathrm{V}_{200}, \mathrm{~V}_{150}$, $\mathrm{V}_{100}, \mathrm{~V}_{90}, \mathrm{~V}_{85}$ per volume coverage, which represents the target volume (TV) covered by $200 \%, 150 \%, 100 \%$, $90 \%$, and $85 \%$ of the prescription dose. Several volumetric parameters have been calculated for the quantitative evaluation of interstitial implants: median and range of coverage index $(\mathrm{CI})$, dose homogeneity index DHI, overdose volume index (ODI), dose non-uniformity ratio (DNR). All these parameters are referred to the BT CTV. Coverage index is the CTV fraction receiving a dose equal to or greater than the reference dose $\left(\mathrm{D}_{\text {ref }}\right)(100 \%$ of dose $)$ and is calculated as $\mathrm{TV}_{\text {Dref/ }}$ $\mathrm{TV}$. The DHI is the CTV fraction receiving a dose in the range of 1 to 1.5 times of the reference dose $\left(\left(\mathrm{TV}_{\mathrm{D}_{\text {ref }}}-\right.\right.$

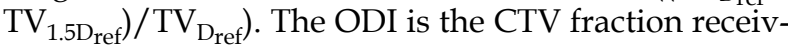
ing a dose equal to or greater than twice the reference dose $\left(\mathrm{TV}_{2.0 \mathrm{Dref}} / \mathrm{TV}_{\mathrm{Dref}}\right)$ [15]. The dose non-uniformity ratio (DNR) is the ratio of the dose volume, which receives a reference dose $(100 \%)$ and that, which receives 1.5 times the reference dose $\left(\mathrm{V}_{150} / \mathrm{V}_{100}\right)$ [16].

\section{Follow-up and toxicity report}

After brachytherapy, all patients were followed every 3 months in the first year, and subsequently, every 4-6 months until the fifth year. During the follow-up, they underwent both clinical and imaging investigations. Acute ( $<6$ months) and late ( $\geq 6$ months) toxicities were reported according to the Common Terminology Criteria for Adverse Events (CTCAE) Version 4.0. [17].

\section{Feasibility and safety criteria assessment}

The main endpoint was to evaluate the feasibility in terms of safety, target coverage, and early toxicity of this procedure. Nine indicators were selected to evaluate the feasibility and safety (Table 1 ). The procedure was considered acceptable if $90 \%$ of the indicators were consistent with the benchmark.

Feasibility indicators were: 1) patients' compliance - percentage of patients who tolerated the procedure and completed it; 2 ) coverage index - median CI calculated as the fraction of the CTV receiving a dose equal to or greater than the reference dose $\left(\mathrm{D}_{\text {ref }}\right)(100 \%$ of dose); 3) rate of correspondence between implant and preplan - percentage of implant realized exactly as preplan; 4) rate of patient with good implant - percentage of patients with implant unmodified after post-implant CT; 5) rate of needles' changes - percentage of needles added or removed after post-implant CT.

Safety indicators instead were: 1) rate of infection - percentage of local and/or systemic infection events observed during and after implants; 2) rate of bleeding and sever pain - percentage of bleeding or sever pain events observed during and after implants; 3) rate of low acute toxicities - percentage of G1-G2 toxicities observed within 6 months according to CTCAE v4; 4) rate of high acute toxicities - percentage of G3G4 toxicities observed within 6 months according to CTCAE v4.

Working group established also the benchmarks for feasibility indicators. Feasibility indicators were: 1) patients' compliance: $>95 \%$; 2) coverage index: $>0,9 ; 3)$ rate of correspondence between implant and pre-plan: $>95 \% ; 4)$ rate of patient with good implant: $>90 \%$; 5) rate of needles' changes: $<5 \%$.

Safety indicators instead were: 1) rate of infection: $<5 \%$; 2 ) rate of bleeding and sever pain: $<15 \%$; 3 ) rate of low acute toxicities: $<30 \%$; 4 ) rate of high acute toxicities: $<5 \%$.

Acute toxicity values were set in line with the recent papers $[8,18,19,20,21]$.

Table 1. Indicators for feasibility and safety

\begin{tabular}{lcc} 
Indicators & Benchmarks & Our study \\
\hline Patients' compliance & $>95 \%$ & $100 \%$ \\
\hline Coverage index & $>0.9$ & 0.94 \\
\hline $\begin{array}{l}\text { Rate of correspondence } \\
\text { between implant and pre-plan }\end{array}$ & $>95 \%$ & $100 \%$ \\
\hline $\begin{array}{l}\text { Rate of patient with good } \\
\text { implant }\end{array}$ & $>90 \%$ & $92 \%$ \\
\hline Rate of needles' changes & $<5 \%$ & $1.9 \%$ \\
\hline Rate of infection & $<5 \%$ & $0 \%$ \\
\hline Rate of bleeding and sever pain & $<15 \%$ & $8 \%$ \\
\hline Rate of low acute toxicities & $<30 \%$ & $27.3 \%$ \\
\hline Rate of high acute toxicities & $<5 \%$ & $0 \%$
\end{tabular}


Table 2. Patients' characteristics

\begin{tabular}{|c|c|}
\hline Factors & $N(\%)$ \\
\hline \multicolumn{2}{|l|}{ Sex } \\
\hline Female & $7(63.6 \%)$ \\
\hline Male & $4(36.4 \%)$ \\
\hline Age mean & 58.6 years (range: $30-78$ ) \\
\hline \multicolumn{2}{|l|}{ Stage } \\
\hline I & $2(18.2 \%)$ \\
\hline II & $3(27.3 \%)$ \\
\hline IIIA & $2(18.2 \%)$ \\
\hline IIIB & $4(36.3 \%)$ \\
\hline \multicolumn{2}{|l|}{ Initial T } \\
\hline $\mathrm{T} 1-\mathrm{T} 2$ & $6(54.5 \%)$ \\
\hline $\mathrm{T} 3-\mathrm{T} 4$ & $5(45.5 \%)$ \\
\hline \multicolumn{2}{|l|}{ Initial N } \\
\hline $\mathrm{N}-$ & $7(63.6 \%)$ \\
\hline $\mathrm{N}+$ & $4(36.4 \%)$ \\
\hline \multicolumn{2}{|l|}{ HIV } \\
\hline Positive & $1(9 \%)$ \\
\hline Negative & $10(91 \%)$ \\
\hline \multicolumn{2}{|l|}{ Histology } \\
\hline Squamous cell carcinoma & $10(91 \%)$ \\
\hline Adenocarcinoma & $1(9 \%)$ \\
\hline Follow-up-median & $\begin{array}{l}25 \text { months } \\
\text { (ranged from } 7 \text { to } 36 \text { months) }\end{array}$ \\
\hline
\end{tabular}

\section{Further analyses}

Moreover, we calculated the response rate, overall survival (OS), and local and distant control rate, which were not included in the main endpoints.

\section{Results}

All anal cancer patients eligible for brachytherapy underwent this novel multiparametric imaging (MR/ CT) guided procedure in our Institute from February 2012 to July 2014. At the time of the study, we treated 11 patients (four men and seven women) and we performed 13 procedures. Three patients presented with major comorbidity: one patient had severe cardiac disease, one patient was HIV positive, and one patient had grade III inguinal-scrotal hernia.

The mean age of the cohort was 58.6 years (range: 30-78). Ten patients had a histological diagnosis of squamous cell anal canal carcinoma (SCACC), whereas one patient presented with adenocarcinoma localized at the anorectal junction. The patient characteristics are reported in Table 2. Three patients underwent conventional 3D-EBRT, among whom, one with a split; the other patients received intensity-modulated radiotherapy (IMRT). The median EBRT CTV1 dose was 51 Gy (range: 44-58.5 Gy).

All patients received mitomycin $\mathrm{C}$ and 5-FU, except one patient who received only 5 -FU because of bad performance status, and one patient who underwent exclusive radiotherapy due to cardiac comorbidities. The younger patient underwent surgical ovarian transposition before EBRT. Only one patient did not complete radiotherapy and the second cycle of chemotherapy because of toxicities and low compliance. In the other patients, we only observed grade 1 gastrointestinal, cutaneous or hematologic toxicities during EBRT. The median overall treatment time (OTT) of radio-chemotherapy was 36 days (ranging from 31 to 59 days). The median overall treatment time of radio-chemotherapy and brachytherapy (OTT-BT) was 95 days (ranging from 59 to 165 days). The median time interval between the end of EBRT and the beginning of BT was 63 days (ranging from 22 to 128 days). Following EBRT, eight patients showed residual tumor in the anal canal. For the first five patients, we used the MUPIT applicator (Elekta). We used the Template set TPS 081 (by GfMmbH, Germany) in all the other cases. The median BT fraction dose was $4 \mathrm{~Gy}$ (range: 3.5-7 Gy). Two patients received the total dose in 2 fractions of $4 \mathrm{~Gy}$. In the HIV patient, the total dose was administered in two fractions in the same day (> 6 hours). The median total BT dose was 4 Gy (range: 3.5-8 Gy). The treatment characteristics are reported in Table 3.

\section{Safety and feasibility}

The analysis of the indicators for the procedure evaluation showed that all markers were consistent with the benchmark (100\%). One patient required local anesthesia during the insertion of the dummy applicator because of severe discomfort (pain). One patient accidentally removed the dummy applicator before the MRI and a new insertion was needed. No other complications appeared during the MRI-procedure as well as during and after the implantation, in terms of severe pain and bleeding. The implant was always performed following the information provided by the pre-plan. No changes regarding the position and the depth of the needles were made before the post-implant CT, so the implants were completely fitted with to the pre-plan catheter arrangement. We observed that one needle overstepped the rectal mucosa in one patient after post-implant CT, thus geometrical analysis allowed to re-adjust it. Consequently, a new CT was performed to check the new needle position.

\section{Dosimetric analysis}

The median TV was 8.7 cc (range: 4.6-36.6 cc). The dosimetric analysis resulted in a median of $\mathrm{V}_{200}$ $24.6 \%, \mathrm{~V}_{150} 53.4 \%, \mathrm{~V}_{100} 93.5 \%$, while the median $\mathrm{V}_{90}$ was $97.6 \%$ and the median $\mathrm{V}_{85}$ was $98.7 \%$. The median 
coverage index $(\mathrm{CI})$, dose homogeneity index (DHI), dose non-uniformity ratio (DNR), and overdose volume index (ODI) were respectively $0.94,0.43,0.56$, and 0.27 . A complete description of dose distribution is reported in Table 4.

We separately analyzed the dosimetric data of two different patient groups according to the type of applicator used, as reported in Table 5. No differences were found in terms of target coverage between the two applicator types. A confirmation from a statistical analysis of significance was not possible because of the small patient sample.

\section{Follow-up}

The median follow-up was 25 months (ranging from 7 to 36 months). Ten patients (91\%) showed a total response after the BT. The patient who did not complete ERT and CT had a persistent disease, thus Miles surgery was performed after 4 months from BT. Ten patients $(91 \%)$ are alive with no evidence of local and distant disease. One patient, died of a pelvic relapse 10 months after BT. Subsequently, this patient underwent total pelvectomy after relapse. The colostomy free survival rate is 81 .

\section{Acute toxicity}

The most common complications were gastrointestinal grade 1, as a matter of fact, we observed fecal incontinence in one patient (CTCAE grade 1) and hemorrhoids in two patients (CTCAE grade 1). No hematological, cutaneous, or genitourinary acute toxicities were observed.

\section{Late toxicity}

We detected anal fibrosis (CTCAE grade 1) in one patient and fecal incontinence in another patient (CTCAE grade 1). Only one patient had grade 2 proctitis. Furthermore, we registered two cases of sacral osteonecrosis, although it was out of the brachytherapy treated area. Both patients had undergone an IMRT treatment. We did not record late toxicities at the time in patient that received 2 fractions in the same day.

\section{Discussion}

This study analysis on the feasibility and the early outcomes of patients treated with radiotherapy $+/-$ chemotherapy, and a novel multiparametric imaging (MR/CT) guided procedure for high-dose-rate (HDR)
Table 3. Treatments' characteristics and toxicities

\begin{tabular}{|c|c|}
\hline Treatment & $N(\%)$ \\
\hline \multicolumn{2}{|l|}{ EBRT technique: } \\
\hline 3D-RT & $3(27.3 \%)$ \\
\hline IMRT & $8(72.7 \%)$ \\
\hline CTV1 ( $T+$ anal canal) dose & 51 Gy (range: 44-58.5 Gy) \\
\hline \multicolumn{2}{|l|}{ Concomitant CT: } \\
\hline$M M C+5-F U$ & $9(81.8 \%)$ \\
\hline 5-FU i.c. alone & $1(9.1 \%)$ \\
\hline No CT & $1(9.1 \%)$ \\
\hline $\begin{array}{l}\text { Residual after EBRT } \\
\text { at 4-6 weeks }\end{array}$ & $8(72.7 \%)$ \\
\hline $\begin{array}{l}\text { Time for residual negativity } \\
\text { after BT median }\end{array}$ & $\begin{array}{c}2.5 \text { months } \\
\text { (range } 2-4 \text { months) }\end{array}$ \\
\hline Fraction dose BT median & 4 Gy (range: 3.5-7 Gy) \\
\hline \multicolumn{2}{|l|}{ Number of fractions: } \\
\hline Single fraction & $9(81.8 \%)$ \\
\hline 2 fraction in the same day & $1(9.1 \%)$ \\
\hline 2 fraction in different days & $1(9.1 \%)$ \\
\hline ERT-BT split median & $\begin{array}{c}63 \text { days (ranging from } 22 \\
\text { to } 128 \text { days) }\end{array}$ \\
\hline $\begin{array}{l}\text { OTT mean (overall treat- } \\
\text { ment time until end ERT) }\end{array}$ & $\begin{array}{c}36 \text { days (ranging from } 31 \\
\text { to } 59 \text { days) }\end{array}$ \\
\hline $\begin{array}{l}\text { OTTB mean (overall treat- } \\
\text { ment time until end BT) }\end{array}$ & $\begin{array}{c}95 \text { days (ranging from } 59 \\
\text { to } 165 \text { days) }\end{array}$ \\
\hline \multicolumn{2}{|l|}{ Acute toxicities: } \\
\hline Gastrointestinal & 3 (3 grade I) (27.3\%) \\
\hline Hematologic & 0 \\
\hline Cutaneous & 0 \\
\hline Genitourinary & 0 \\
\hline \multicolumn{2}{|l|}{ Late toxicities: } \\
\hline Gastrointestinal & 3 (2 grade I; 1 grade II) (27.3\%) \\
\hline Hematologic & 0 \\
\hline Cutaneous & 0 \\
\hline Genitourinary & 0 \\
\hline
\end{tabular}

EBRT - external beam radiotherapy, 3D-RT - 3D conformal radiotherapy, IMRT - intensity modulated radiotherapy, CTV - clinical target volume, $E R T$ - external radiation therapy, Concomitant CT - concomitant chemotherapy, $M M C$ - mitomycin C, 5-FU - 5-fluorouracil, BT - brachytherapy, OTT - overall treatment time, OTTB - overall treatment time until end BT

Table 4. Dosimetric analysis

\begin{tabular}{|c|c|c|c|c|c|c|c|c|c|c|}
\hline & $V_{200}$ & $V_{150}$ & $V_{100}$ & $\mathrm{~V}_{90}$ & $\mathrm{~V}_{85}$ & TV & $\mathrm{Cl}$ & DHI & DNR & ODI \\
\hline Mean & $28.07 \%$ & $53.11 \%$ & $93.04 \%$ & $97.56 \%$ & $98.60 \%$ & 11.9 & 0.92 & 0.43 & 0.56 & 0.29 \\
\hline Median & $24.62 \%$ & $53.43 \%$ & $93.5 \%$ & $97.63 \%$ & $98.74 \%$ & 8.7 & 0.94 & 0.43 & 0.56 & 0.27 \\
\hline Range & $4.7-61.6 \%$ & $23.11-85.23 \%$ & $86.64-97.64 \%$ & $94.02-99.84 \%$ & $96.09-100 \%$ & $4.6-36.6$ & $0.74-0.99$ & $0.14-0.68$ & $0.31-0.85$ & $0.16-0.62$ \\
\hline
\end{tabular}

$V_{200}, V_{150}, V_{100}, V_{90}, V_{85}$ represents the TV covered by $200 \%, 150 \%, 100 \%, 90 \%$, and $85 \%$ of the prescription dose, TV - target volume, Cl-coverage index, DHI- dose homogeneity index, DNR - dose non-uniformity ratio, ODI - overdose volume index 
Table 5. Dosimetry in different applicators

\begin{tabular}{lcccc} 
& $\mathrm{V}_{150}$ & $\mathrm{~V}_{100}$ & $\mathrm{~V}_{90}$ & $\mathrm{~V}_{85}$ \\
\hline MUPIT & & & & \\
\hline Mean & $55.46 \%$ & $92.50 \%$ & $97.59 \%$ & $98.42 \%$ \\
\hline Median & $58.79 \%$ & $93.45 \%$ & $96.78 \%$ & $98.74 \%$ \\
\hline TPS 081 & & & & \\
\hline Mean & $51.15 \%$ & $93.31 \%$ & $97.55 \%$ & $98.69 \%$ \\
\hline Median & $49.43 \%$ & $92.81 \%$ & $97.66 \%$ & $98.83 \%$
\end{tabular}

$V_{150}, V_{100}, V_{90}, V_{85}$ represents the target volume covered by $150 \%, 100 \%, 90 \%$, and $85 \%$ of the prescription dose; MUPIT - Martinez Universal Perineal Interstitial Template (Elekta $)$; TPS 081 the template set TPS 081 (by GFMmbH $\left.H^{\oplus}\right)$

image adapted brachytherapy (IABT) in anal canal cancer. Since all indicators are in agreement with the benchmarks, we can consider this procedure feasible and safe. Standard curative treatment of anal cancer includes EBRT with concomitant chemotherapy and achieves high local tumor control (LC), overall survival (OS) rates, as well as high sphincter preservation rates [3, 22, 23, 24, 25, 26]. Brachytherapy has an important role as a local dose escalation (boost) in the treatment of anal cancer [8].

As the 2014 ESMO-ESSO-ESTRO Anal Cancer clinical practice guidelines state, brachytherapy is a highly conformal treatment, which can treat the primary tumor, sparing the surrounding normal tissues, and the contra-lateral mucosa and sphincter. Exclusive curative $\mathrm{BT}$ is not recommended, although it may be applicable as a boost following radiochemotherapy [1]. However, improvements in local tumor control by radiochemotherapy with additional brachytherapy, may be limited by side-effects, such as sphincter necrosis, resulting in incontinence, or stenosis. These complications may lead to colostomy [27] but are influenced by the different types of BT boost and are less frequent nowadays due to technological developments (e.g. use of image guidance).

For example, Berger et al. reported a five-year local control rate of $59 \%$ and a colostomy rate of $33 \%$ in a retrospective analysis of 69 patients treated with 20 Gy interstitial LDR-BT after 40 Gy EBRT. In the ACT I cancer trial [28], a total of 577 patients were randomly assigned RT alone without a split or combined radiochemotherapy, using 5-FU and mitomycin C. Afterwards, six-week responders received a boost using either additional external beam radiotherapy (EBRT) (15 Gy) or LDR ${ }^{192}$ Ir implant (25 Gy). The study reported $38 \%$ decrease of anal cancer death risk among patients undergoing some form of boost, either by implant or EBRT boost. There was a trend of reduced risk of loco-regional failure for patients given the implant boost, compared to the administration of no boost. An improved outcome for patients receiving radiochemotherapy was detected among the implanted patients, yet observed to a lesser extent, in those who received EBRT boost. Radionecrosis was reported in $8 \%$ of boosted patients, compared with $0 \%$ in non-boosted patients $(p=0.03)$. Furthermore, there were more reports of ulcers/radionecrosis for the subgroup who received boost by implant (14\%) compared with the EBRT boost subgroup $(6 \% ; p=0.003)$. In the ACT I study, patients received a BT boost with LDR linear source brachytherapy, which cannot allow the target dose to be optimized as HDR and PDR techniques. Indeed, the positioning of brachytherapy needles was performed in the past by considering the tumor extent and target volume by clinical findings and not by imaging. Moreover, treatments were performed without the use of target dose optimizing possibilities of a stepping source. Also, the ESMO-ESSO-ESTRO Anal Cancer clinical practice guidelines state that double-plane or volume implants may be related to the risk of late necrosis and radiation proctitis, hence they point out the advantage in the use of computerized 3D image-based treatment planning, which allows optimal dose distribution [1].

Therefore, for the time being, many authors talk about a new kind of brachytherapy that can be modulated by the use of IMRT principles, IMBT, and image guiding dose-volume optimization in IGBT - IABT [29].

Image adapted brachytherapy leads to a better target volume definition with a higher quality of implants, resulting in improved normal tissue preservation, and decreasing acute and late toxicity as well improving local control. Today, IABT has become feasible thanks to the remarkable progress of three dimensional (3D) imaging, rapidly increased speed and capacity of computers, and the sophisticated techniques in treatment planning. The dose modulation potential paired with biological target imaging information has paved the way for a new era [29].

Compared to traditional approaches, IABT with imaging compatible applicators, has allowed a more accurate delineation of GTV and CTV, as well as healthy (critical) organs. It has had a direct impact on treatment planning, particularly by optimizing the isodoses also to the target sub-volumes. Furthermore, using traditional dosimetry systems, the prescription dose is related to the geometry of the implant and not to the individual target volume. In modern IABT procedures, both treatment planning and plan evaluation must be based on an actual 3D volume of the PTV and of OARs.

Endoanal ultrasound (EAUS) is usually the first choice in anal canal BT for implant guidance. Indeed, EAUS is the most frequently used imaging method for exact guidance of anal canal interstitial radiotherapy. Compared to conventional brachytherapy, EAUS-guided brachytherapy decreases the side effects [9]. Several studies have demonstrated that endosonography should be preferred when evaluating the tumor extension [30,31], surpassing digital rectal examination, and it is also significant in assessing the position of brachytherapy needles. In his pilot study, Doniec [9] demonstrated that EAUS-guided BT for anal cancer is safe in terms of side effects compared to conventional brachytherapy. Furthermore, the use 
of this technology combined with brachytherapy improves local tumor control and minimizes morbidity in patients with anal carcinoma.

Christensen et al. investigated the possibility of using 3D endosonography for BT surpassing ordinary 2D endosonography. In his preliminary study, he underlines that three-dimensional endosonography guidance of interstitial brachytherapy in anal carcinoma optimizes the implant procedure and provides better information for dose planning and possibly improved local tumor control; although it is necessary to have long-term follow-up results [32]. Moreover, under ultrasound control, the needles are implanted directly into the tumor or tumor bed and real time treatment planning is possible [7]. Endoanal ultrasound is relatively cheap, safe, and well tolerated despite the limitation of its field of action. It is also operator-dependent and unable to assess stenotic tumors [33]. MRI-based implants are a possible alternative in order to overcome such limits. Indeed, thanks to contrast and spatial resolution, MRI imaging is an excellent means to assess the tumor size, local extent and spreading, and invasion to adjacent organs. In addition, it is more accurate in detecting nodal involvement, thus facilitating treatment planning [1, 10,33]. Compared to EAUS, MRI is able to distinguish edema and scar tissue from persistent or recurrent tumor. Furthermore, it helps to evaluate clinical response after radiochemotherapy avoiding unnecessary biopsies [33, 34].

The most exploited sequences are generally T2weighted that provide high spatial and contrast resolution, offering detailed anatomical imaging and showing the relationship of the tumor with the anal sphincter complex [33].

Furthermore, DWI can be relevant in anal canal cancer in differentiating suspected small residual/recurrent treatment-related changes and in identifying pelvic lymph-nodes [34]. This feature can be fundamental in assessing the therapeutic pathway and indication to perform BT. In recent years, however, there have been substantial improvements in MRI protocols with a special focus on the assessment of functional tissue characteristics, such as perfusion or metabolism. The use of these functional imaging techniques combines information on detailed anatomy with tumor biology, providing extensive information on tumor heterogeneity and its changes as a result of treatment. For the time being, there is some experience with MRI-perfusion in different diseases, such as breast and prostate cancer, renal rejection, brain disease, and liver tumors $[35,36]$. Also, in anal cancer, sequences of MRI perfusion, identifying the vascular tissue, and subsequently the hypoxic areas, could be used to assess the prognosis but also to evaluate radiosensitivity, and thus, foster the dose modulation. On the other hand, a procedure using MRI is time consuming and expensive compared to the US, although it has become the gold standard in the brachytherapy site, where its efficacy has been demonstrated compared to other techniques. In the future, we could demonstrate this concept with enhanced software. Nowadays, MR-IABT is routinely employed for gynecological cancers. As a matter of fact, MRI is the reference imaging tool for brachytherapy of cervix cancer [37, 38]. Advantages of MRI imaging in gynecological BT that may lead to improved patient outcome, irrespective of the dose rate, include avoidance or early detection of a uterine perforation, ensuring target coverage, and avoiding excessive dose to the OAR. Moreover, 3D image-based treatment planning in cervical cancer is recommended in published guidelines of the Gynecological (GYN) GEC-ESTRO Working Group (GEC-ESTRO) [39, 40]. In 2008, Christensen et al. and, in 2014, Niehoff, reported the possible use of MRI in interstitial BT of the anus. The stated limitations include the possibility of having an open MRI machine, and the necessity of MRI compatible templates and needles $[8,32]$. Following the example of gynecological BT we were able to implement a BT based on MR and CT imaging, thanks to an MRI compatible dummy applicator in the anal canal, which stretches the anal cavity to better evaluate the disease. In this way, an improved treatment plan can favor a good treatment. Our protocol established a revaluation after 4-6 weeks. This time duration is considered too long for some authors but it allows us to identify the subset of patients that need BT boost. Moreover, particularly with IMRT technique, we delivered a higher EBRT dose compared to other BT boost experiences published. Moreover, other authors reported a protocol that established BT after 4-6 weeks $[18,41]$.

Regarding the dosimetric analysis, we obtained an optimal median coverage index (0.94) and median $\mathrm{V}_{100}$ (93.5\%). Concerning other parameters calculated, the median DHI was 0.43 and the median DNR was 0.56 . In the analysis published in 2014 by Kapoor et al. [19], the DHI was 0.83 and the DNR was 0.37 . In another analysis published in the same year by Falk et al. [18], these parameters were 0.58 and 0.38 , respectively. Major et al. considered that larger target volume involves a longer active length, and the increase of active length improves the dose uniformity and the optimal dosimetry. Our parameters can be justified by the median of the treatment volume. Indeed, in this analysis the median TV is 8.7 cc., which is much smaller than in other authors (in the paper by Kapoor et al., the mean TV is $17.7 \pm 4.98 \mathrm{cc}$, and in the paper by Falk et al., the median TV is $22.3 \mathrm{cc}$ ) $[42,43]$. Therefore, the implant geometry is applicator-dependent. Although our treatment plans presented a median $V_{150}$ of $53 \%$, we did not report any major toxicities in terms of sphincter necrosis.

Despite some limitations in our study (e.g. the monocentric data collection and the short follow-up), our preliminary results show that MR/CT-guided BT appears feasible and safe with good patient compliance, and without complications during and after the implant procedure. These early results are comparable with other series, which used EAUS guidance techniques. Although the use of MRI in the anal BT presents some limitations (such as costs, time required, and staff training), it could be the preferable choice for the modulation of the target dose according to perfu- 
sion and DWI information (biological image guided brachytherapy). Indeed, the metabolic information could help to deliver a very high dose only to a small volume (metabolically active areas, with increased radioresistance or areas of suspected hypoxia), in order to reduce the high dose volumes as well the dose on the organs at risk, resulting in lower toxicity and increased local control. However, the procedure is expensive and requires a dedicated interdisciplinary team. Furthermore, the advantages compared to EAUS guided techniques have to be demonstrated in the future. The present study shows that it is feasible to perform an MR/CT-guided brachytherapy for anal canal cancer, and in the future, this could allow to investigate the correlation between prognosis and dose modulation, based on MRI functional information.

We are also testing a total MRI compatible applicator that may simplify the procedure because, by using MRI compatible instruments, we can perform the implantation, simulation, and BT procedure at the same time. This procedure could also permit a reduction of the overall treatment time.

\section{Conclusions}

Brachytherapy is a possible option in anal cancer radiotherapy to perform the boost to complete EBRT. Magnetic resonance imaging can also have biological advantages compared to the US. Our results suggest that the multiparametric MR/CT-IABT for anal cancer is feasible and safe. This new approach paves the way to prospective comparison studies between MRI and USBT in anal canal cancer.

\section{Disclosure}

Authors report no conflict of interest.

\section{References}

1. Glynne-Jones R, Nilsson PJ, Aschele C e al. Anal cancer: ESMO-ESSO-ESTRO clinical practice guidelines for diagnosis, treatment and follow-up. Radiother Oncol 2014; 111: 330-339.

2. Epidermoid anal cancer: results from the UKCCCR randomized trial of radiotherapy alone versus radiotherapy, 5-fluorouracil, and mitomycin. UKCCCR Anal Cancer Trial Working Party. UK Co-ordinating Committee on Cancer Research. Lancet 1996; 348: 1049-1054.

3. Wang Y, Cummings B, Catton P et al. Primary radical external beam radiotherapy of rectal adenocarcinoma: long term outcome of 271 patients. Radiother Oncol 2005; 77: 126-132.

4. Hannoun-Levi JM, Ortholan C, Resbeut M et al. High-dose split-course radiation therapy for anal cancer: outcome analysis regarding the boost strategy (CORS-03 study). Int J Radiat Oncol Biol Phys 2011; 80: 712-720.

5. Milano MT, Jani AB, Farrey KJ et al. Intensity-modulated radiation therapy (IMRT) in the treatment of anal cancer: toxicity and clinical outcome. Int J Radiat Oncol Biol Phys 2005; 63: 354-361.

6. Menkarios C, Azria D, Laliberté B et al. Optimal organ sparing intensity-modulated radiation therapy (IMRT) regimen for the treatment of locally advanced anal canal carcinoma: a comparison of conventional and IMRT plans. Radiat Oncol 2007; 2: 41.
7. Löhnert M, Doniec JM, Kovács G et al. New method of radiotherapy for anal cancer with three-dimensional tumor reconstruction based on endoanal ultrasound and ultrasound-guided afterloading therapy. Dis Colon Rectum 1998; 41: 169-176.

8. Niehoff $P$, Kovàcs G. HDR brachytherapy for anal cancer. J Gastrointest Oncol 2014; 5: 218-222.

9. Doniec JM, Schniewind B, Kovács G et al. Multimodal therapy of anal cancer added by new endosonographic guided brachytherapy. Surg Endosc 2006; 20: 673-678.

10. Goh V, Gollub FK, Liaw J et al. Magnetic resonance imaging assessment of squamous cell carcinoma of the anal canal before and after chemoradiation: can MRI predict for eventual clinical outcome? Int J Radiat Oncol Biol Phys 2010; 78: 715-721.

11. DeVries AF, Piringer G, Kremser C et al. Pretreatment Evaluation of Microcirculation by Dynamic Contrast-Enhanced Magnetic Resonance Imaging Predicts Survival in Primary Rectal Cancer Patients. Int J Radiat Oncol Biol Phys 2014; 90: 1161-1167.

12. Dimopoulos JC, Schard G, Berger D et al. Systematic evaluation of MRI findings in different stages of treatment of cervical cancer: potential of MRI on delineation of target, pathoanatomic structures, and organs at risk. Int J Radiat Oncol Biol Phys 2006; 64: 1380-1388.

13. Valentini V, Maurizi F, Tagliaferri L et al. Spider: managing clinical data of cancer patients treated through a multidisciplinary approach by a palm based system. Ital J Public Health JPH 2008; 6.

14. Fokdal L, Tanderup K, Nielsen SK et al. Image and laparoscopic guided interstitial brachytherapy for locally advanced primary or recurrent gynaecological cancer using the adaptive GEC ESTRO target concept. Radiother Oncol 2011; 100: 473-479.

15. Meertens H, Borger J, Steggerda $M$ et al. Evaluation and optimization of interstitial brachytherapy dose distribution. In: Brachytherapy from radium to optimization. Mould RF, Battermann JJ, Martinez AA, Speiser BL (eds.). Nucletron International, Veenendaal 1994; 300-306.

16. Saw CB, Suntharalingam N, Wu A. Concept of dose nonuniformity in interstitial brachytherapy. Int J Radiat Oncol Biol Phys 1993; 26: 519-527.

17. U.S. Department of Health and Human Services, National Cancer Institute, Common Terminology Criteria for Adverse Events (CTCAE) Version 4.0, Published: May 28, 2009 (v4.03: June 14, 2010).

18. Falk AT, Claren A, Benezery K et al. Interstitial high-dose rate brachytherapy as boost for anal canal cancer. Radiat Oncol 2014; 6: 240.

19. Kapoor R, Kholsa D, Shukla AK et al. Dosimetric and clinical outcome in image-based high-dose-rate interstitial brachytherapy for anal cancer. Brachytherapy 2014; 13: 388-393.

20. Lòpez Guerra JL, Lozano AJ, Pera J et al. Twenty-year experience in the management of squamous cell anal canal carcinoma with interstitial brachytherapy. Clin Transl Oncol 2011; 13: $472-479$.

21. De Bari B, Lestrade L. Chekrine T et al. Should the treatment of anal carcinoma be adapted in the elderly? A retrospective analysis of acute toxicities in a French center and a review of the literature. Cancer Radiother 2012; 16: 52-57.

22. Epidermoid anal cancer: results from the UKCCCR randomised trial of radiotherapy alone versus radiotherapy, 5-fluorouracil, and mitomycin. UKCCCR Anal Cancer Trial Working Party. UK Co-ordinating Committee on Cancer Research. Lancet 1996; 348: 1049-1054.

23. Deo SV, Schukla NK, Raina V et al. Organ-preserving multimodality management of squamous cell carcinoma of anal canal. Indian J Gastroenterol 2005; 24: 201-204. 
24. Peiffert D, Giovannini M, Ducreux M et al. High-dose radiation therapy and neoadjuvant plus concomitant chemotherapy with 5-FU and cisplatin in patients with locally advanced squamous cell anal cancer: final results of phase II study. Ann Oncol 2001; 12: 397-404

25. Smith AJ, Whelan P, Cummings BJ et al. Management of persistent or locally recurrent epidermoid cancer of the anal canal with abdominoperineal resection. Acta Oncol 2001; 40: 34-36.

26. Nigro ND. An evaluation of combined therapy for squamous cell cancer of the anal canal. Dis Colon Rectum 1984; 27: 763-766.

27. Peiffert D, Bey P, Pernot $\mathrm{M}$ et al. Conservative treatment by irradiation of epidermoid cancers of the anal canal: prognostic factors of tumoral control and complications. Int J Radiat Oncol Biol Phys 1997; 37: 313-324.

28. Glynne-Jones R, Sebaq-Montefiore D, Adams R et al. "Mind the gap" - the impact of variations in the duration of the treatment gap and overall treatment time in the first UK Anal Cancer Trial (ACT I). Int J Radiat Oncol Biol Phys 2011; 81: 1488-1494.

29. Meyer JE, Brocks C, Maune S et al. Brachytherapy for the treatment of head and neck cancer. HNO 2010; 58: 947-958.

30. Magdeburg B, Fried M, Meyenberger C. Endoscopic ultrasonography in the diagnosis, staging and follow-up of anal carcinoma. Endoscopy 1999; 31: 359-364.

31. Roseau G, Palazzo L, Colardelle P et al. Endoscopic ultrasonography in the staging and follow-up of epidermoid carcinoma of the anal canal. Gastrointest Endosc 1994; 40: 447-450.

32. Christensen AF, Nielsen BM, Engelholm SA et al. Three-dimensional endoluminal ultrasound-guided interstitial brachytherapy in patient with anal cancer. Acta Radiol 2008; 49: 132-137.

33. Parikh J, Shaw A, Grant LA et al. Anal carcinomas: the role of endoanal ultrasound and magnetic resonance imaging in staging, response evaluation and follow-up. Eur Radiol 2011; 21: 776-785.

34. Gourtsoyianni S, Goh V. MRI of anal cancer: assessing response to definitive chemoradiotherapy. Abdom Imaging 2014; 39: 2-17.

35. Beets-Tan RG, Beets GL. MRI for assessing and predicting response to neoadjuvant treatment in rectal cancer. Nature Rev Gastroenterol Hepatol 2014; 11: 480-488.

36. Khalifa F, Soliman A, El-Baz A et al. Models and methods for analyzing DCE-MRI: a review. Med Phys 2014; 41: 124301.

37. Petric P, Mohammed-Al-Hammadi N. MRI findings at image guided adaptive cervix cancer brachytherapy: radiation oncologist's perspective. J Contemp Brachytherapy 2014; 6: 215-222.

38. Pötter R, Georg P, Dimopoulos JC et al. Clinical outcome of protocol based image (MRI) guided adaptive brachytherapy combined with 3D conformal radiotherapy with or without chemotherapy in patients with locally advanced cervical cancer. Radiother Oncol 2011; 100: 116-123.

39. Haie-Meder C, Pötter R, Van Limbergen E et al. Recommendations from Gynaecological (GYN) GEC-ESTRO Working Group (I): concepts and terms in 3D image based 3D treatment planning in cervix cancer brachytherapy with emphasis on MRI assessment of GTV and CTV. Radiother Oncol 2005; 74: 235-245.

40. Pötter R, Haie-Mader C, Van Limbergen E et al. Recommendations from gynaecological (GYN) GEC-ESTRO Working Group: (II): concepts and terms of 3D imaging, radiation physics, radiobiology, and 3D dose volume parameters. Radiother Oncol 2006; 78: 67-77.

41. Papillon J, Montbarbon JF. Epidermoid carcinoma of the anal canal: a series of 276 cases. Dis Colon Rectum 1987; 30: 324-333.

42. Major T, Polgár C, Fodor J et al. Conformality and homogeneity of dose distributions in interstitial implants at idealized taget volumes: a comparison between the Paris and dosepoint optimized systems. Radiother Oncol 2002; 62: 103-111.
43. Major T, Polgàr C, Somogyi A et al. Evaluation of the dose uniformity for double-plane high dose rate interstitial breast implants with the use of dose reference points and dose non-uniformity ratio. Radiother Oncol 2000; 54: 213-220. 